\title{
Acromegaloid facial appearance (AFA) syndrome: report of a second family
}

\author{
Bruno Dallapiccola, Leopoldo Zelante, Laura Accadia, Rita Mingarelli
}

\begin{abstract}
A family is reported in which the 'acromegaloid facial appearance' (AFA) phenotype was segregating through two generations. The five affected persons showed a striking resemblance to the patients previously reported, including progressively coarse acromegaloid-like facial appearance, narrow palpebral fissures, bulbous nose, and thickening of the lips and intraoral mucosa, resulting in exaggerated rugae and frenula. These patients also had increased birth weight and dull mentality. It is unclear if the differences between the two families mark distinct syndromes or simply extend the AFA phenotype.
\end{abstract}

The acromegaloid facial appearance (AFA) syndrome has been described in a large kindred in which affected persons spanning at least five generations had progressively coarse facial changes and thickening of the lips and intraoral mucosa. ${ }^{1}$ Despite wide phenotypic variability, this autosomal dominant mutation showed complete penetrance. Pachydermoperiostosis, Ascher syndrome, and multiple neuroma syndrome were considered in the differential diagnosis.

Since the original study in 1985 no other case of AFA syndrome has been published. Here, we report on a family in which an AFAlike phenotype was transmitted from an affected mother to four of her five children.

\section{Case reports}

This family came to our attention in 1984, when the mother was admitted to our hospital for mild anaemia and suspected hypothyroidism. At that time all family members were evaluated and transmission of an unreported autosomal dominant syndrome from the mother to four of her five children was suspected (fig 1). The family was re-evaluated at the end of 1990 when a diagnosis of AFA syndrome was suspected.

The proband, a 52 year-old woman, was the only term product of healthy, non-consanguineous parents. Birth weight was $4000 \mathrm{~g}$. Her perinatal period was unremarkable and her early developmental milestones were reported to be within normal limits. No learning difficulties were noticed during her school years. She married at 22 years. A photograph taken at that time shows only minimal facial signs, including thickened upper eyelids leading to narrow palpebral fissures and a slight swelling of the lower lip (fig 2).

During the years 1963 to 1979 she became pregnant 14 times. Eight pregnancies were terminated. The last pregnancy resulted in the premature delivery of stillborn dizygotic twins. The five pregnancies that came to term resulted in three females and one male with AFA syndrome. One boy is normal. Progressive changes in the face and extremities became apparent before the age of 30 years.

When evaluated by us at 52 years (fig 2 ), her height was $160 \mathrm{~cm}$ and she weighed $63 \mathrm{~kg}$. She had a flat and mildly sloping forehead. The facial skin was thickened, especially around the eyes, leading to narrowed palpebral fissures. The eyebrows were bushy with synophrys. There was hypertelorism, with increased intercanthal distance ( $>97$ th centile). The nose was bulbous with thick nostrils and the nasal septum extended below the nasal alae. The philtrum was slightly elongated. The lips and intraoral mucosa were thickened with exaggerated frenula (fig 3 ) and rugae and a large tongue. There was also mild micrognathia. The ears were normal. The hands were large (fig 3) with hyperextensible metacarphophalangeal and interphalangeal joints. Dermatoglyphics were normal with 10 whorls on the fingertips and proximally located axial triradii.

She had a normal but dull mentality. A general slowing of all intellectual functions, including speech, was apparent. Routine
Biology, Division of Vergata University, Via Ramazzini, 00151 Roma, Italy.

R Mingarelli

Divisions of Medical Genetics and Gastroenterology, CSS Hospital, Institute of Research, 71013 San Giovanni Rotondo, Italy. L Zelante

L Accadia

Correspondence to Dr Dallapiccola.

Received 11 July 1991. Revised version accepted 23 September 1991. 

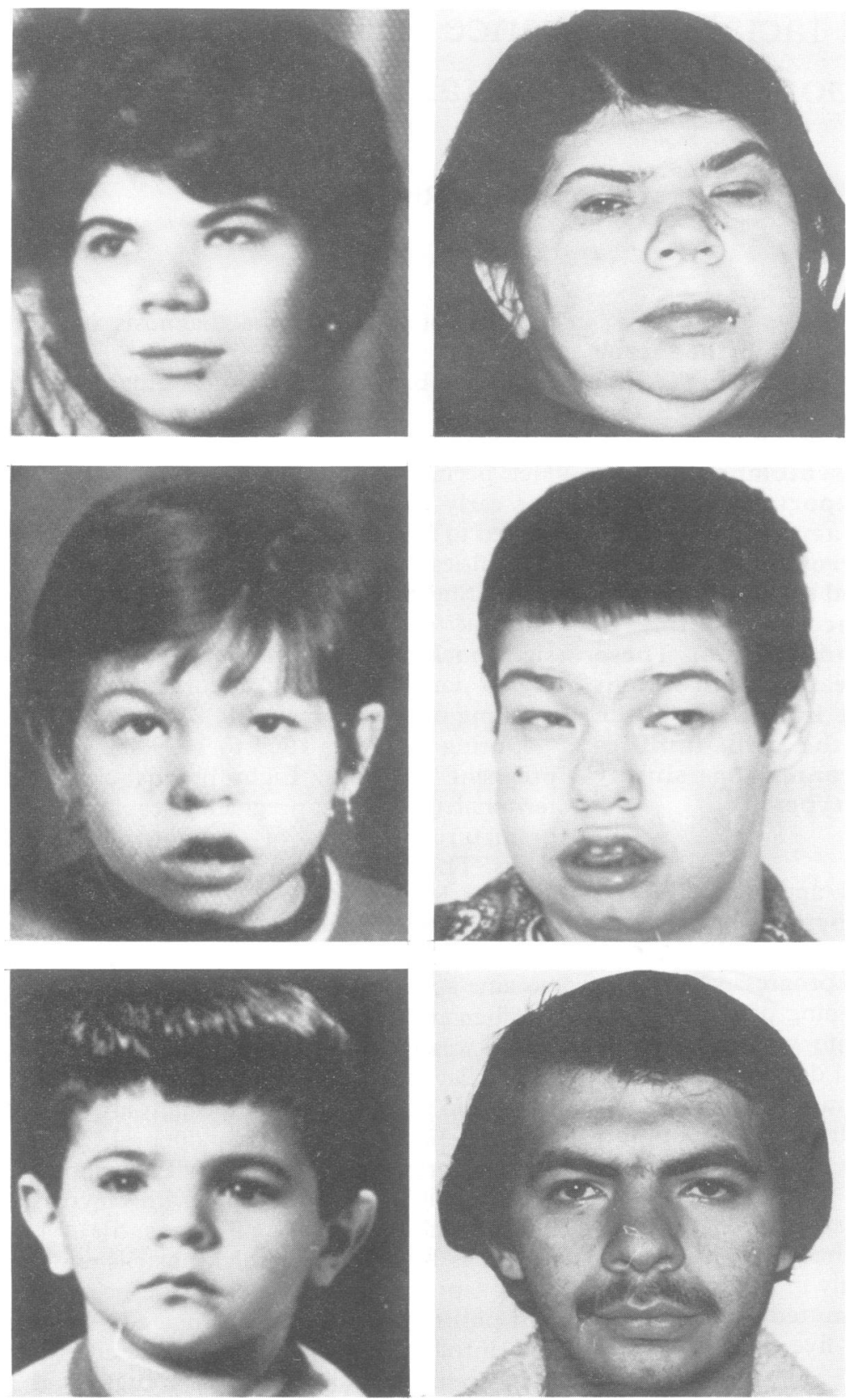

Figure 2 The mother aged 22 and 52 years (top row), and the first sib (middle row) and the second sib (bottom row) in childhood and aged 27 and 25 years.

Comparison of clinical features in the five patients of the present family and in the AFA syndrome patients.

\begin{tabular}{lcccccc}
\hline & \multicolumn{7}{c}{ Present family } & AFA \\
\cline { 2 - 6 } Features & II.2 & III.2 & III.3 & III.5 & III.8 & family \\
\hline Increased birth weight (g) & 4000 & 4200 & 6400 & 5500 & 4000 & - \\
Thick cranial vault & + & $?$ & + & + & $?$ & $?$ \\
Flat/sloping forehead & + & + & + & + & + & - \\
Synophrys & + & + & + & $+/$ & $+/$ & - \\
Increased intercanthal distance & $>97 \mathrm{c}$ & + & $>97 \mathrm{c}$ & $>97 \mathrm{c}$ & $>97 \mathrm{c}$ & $(+)$ \\
Thick upper eyelid & + & + & + & + & + & + \\
Bulbous nose & + & + & + & + & + & + \\
Thick nasal alae & + & + & + & + & + & + \\
Prominent philtrum & + & + & + & + & + & $(+)$ \\
Prominent lips & + & + & + & $+/-$ & + & + \\
Exaggerated rugae and frenula & + & + & + & + & + & + \\
Tongue (Large/Furrowed) & L & L & F & F & L & + \\
Micrognathia & + & + & + & + & + & + \\
Large hands & + & + & + & + & + & + \\
Short 5th metacarpals & + & $?$ & + & + & $?$ & $?$ \\
Hyperextensible joints & + & + & + & + & + & + \\
Soft tissue swelling & + & + & + & + & + & + \\
Dull mentality & + & Severe & + & + & + & - \\
& & MR & & & & \\
\hline
\end{tabular}

laboratory investigations, thyroid function, growth hormone assay, and karyotype analysis were normal. A radiographic skeletal survey showed a thickened and dysostotic cranial vault, enlarged distal radial epiphyses, and short fifth metacarpals.

The first four sibs, three females and one male, showed a striking resemblance to their mother (figs 2, 3, and 4); their ages ranged between 20 and 27 years. The youngest child, a 14 year old boy, was clinically normal.

The affected sibs had increased birth weight (range 4000 to $6400 \mathrm{~g}$ ) and dysmorphic features affecting the face and extremities (table). According to the mother the four children developed facial coarseness from early childhood. All were in good health and had normal height and weight. All required special class placements suggesting that dull mentality is a feature of AFA syndrome in this family. The first affected daughter is severely mentally retarded. According to the Wechsler Adult Intelligence Scale - Revised, IQ was 48. From the age of 16 years she has been in an institution for the mentally retarded.

Thyroid function and growth hormone assays were carried out in the four affected sibs and the results were within normal limits.

\section{Discussion}

In this kindred, five persons in two generations have the features of the syndrome originally described by Hughes et al. ${ }^{1}$ The most striking characteristics in the affected subjects are progressive, coarse facial changes and large hands with doughy skin, but without clubbing, reminiscent of acromegaly, thickened upper eyelids, leading to narrow palpebral fissures, bulbous nose, thickened lips with overgrowth of the intraoral mucosa resulting in exaggerated rugae and frenula, and a large or furrowed tongue. In published photographs from the original family, the eyebrows appeared strikingly highly arched. This was not a feature in our patients. All five affected persons studied by us had increased birth weight. The only unaffected boy in the sibship had a normal birth weight.

Hughes et $a l^{1}$ found seizures and learning difficulties in only one of the 13 affected persons examined in their family. Since all other affected subjects had normal intelligence it was concluded that mental impairment was not a feature of AFA syndrome. Mental dullness was present in four of the five patients in our family and severe mental retardation in one. Thus, there are marked similarities between these two families, but there are also significant differences which could argue against them being one and the same entity (table). If the AFA syndrome is segregating in the present family, then our observation extends the AFA phenotype, and also questions the appropriateness of the 'acromegaloid facial appearance' designation for this condition. Progressive facial coarseness is a feature of acromegaly, in which bony deformity of the skull becomes apparent. Consistent features are overgrowth of the frontal, malar, and nasal bones and 
Figure 3 Exaggerated oral frenula in the mother and third sib (top row), fissured and furrowed tongue in the second and third sibs (middle row), and enlarged hands with tapered fingers in the mother and first daughter (bottom row).
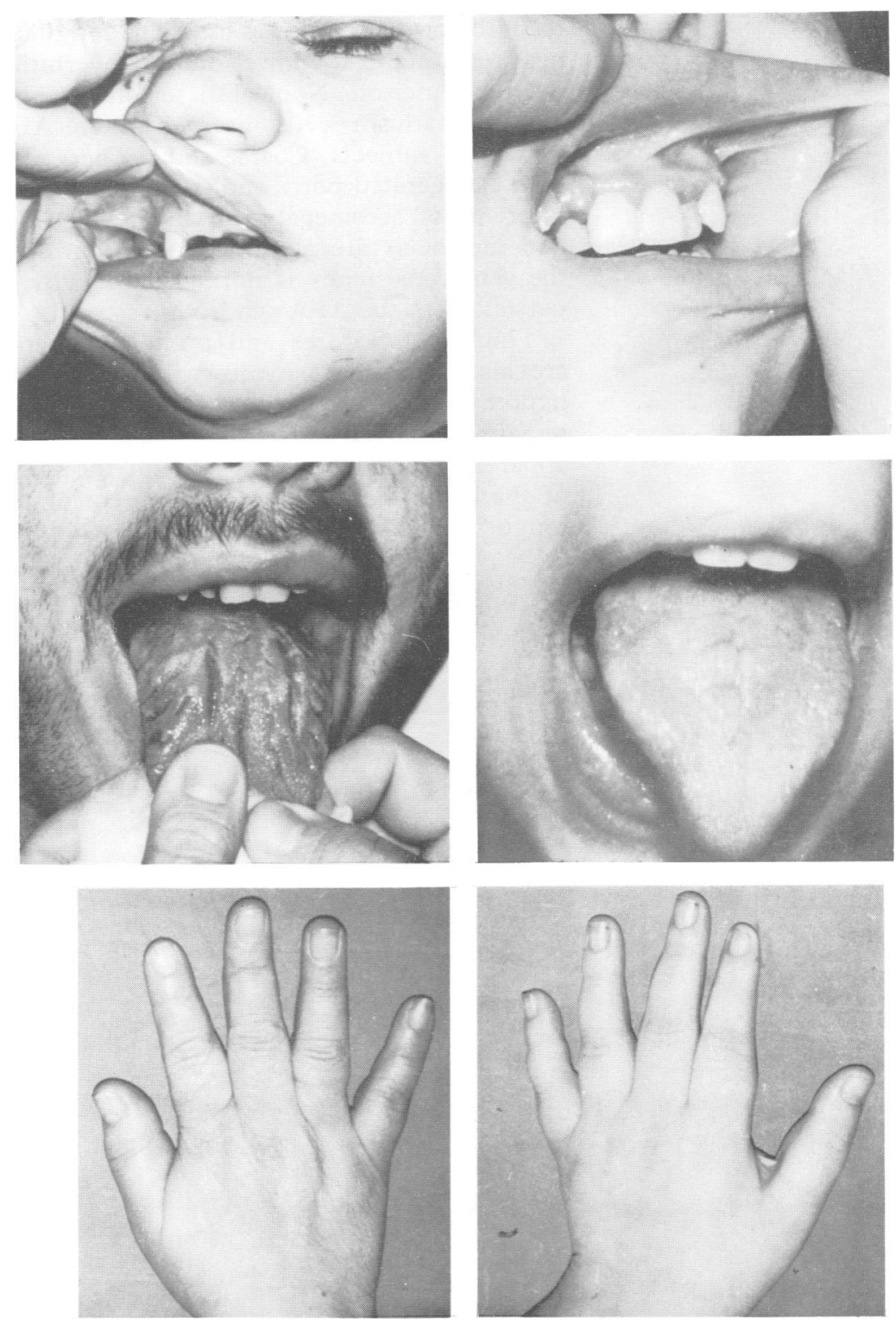

Figure 4 The third sib (top row) aged 16 and 22 years, and the fourth sib (bottom row) aged 13 and 19 years.
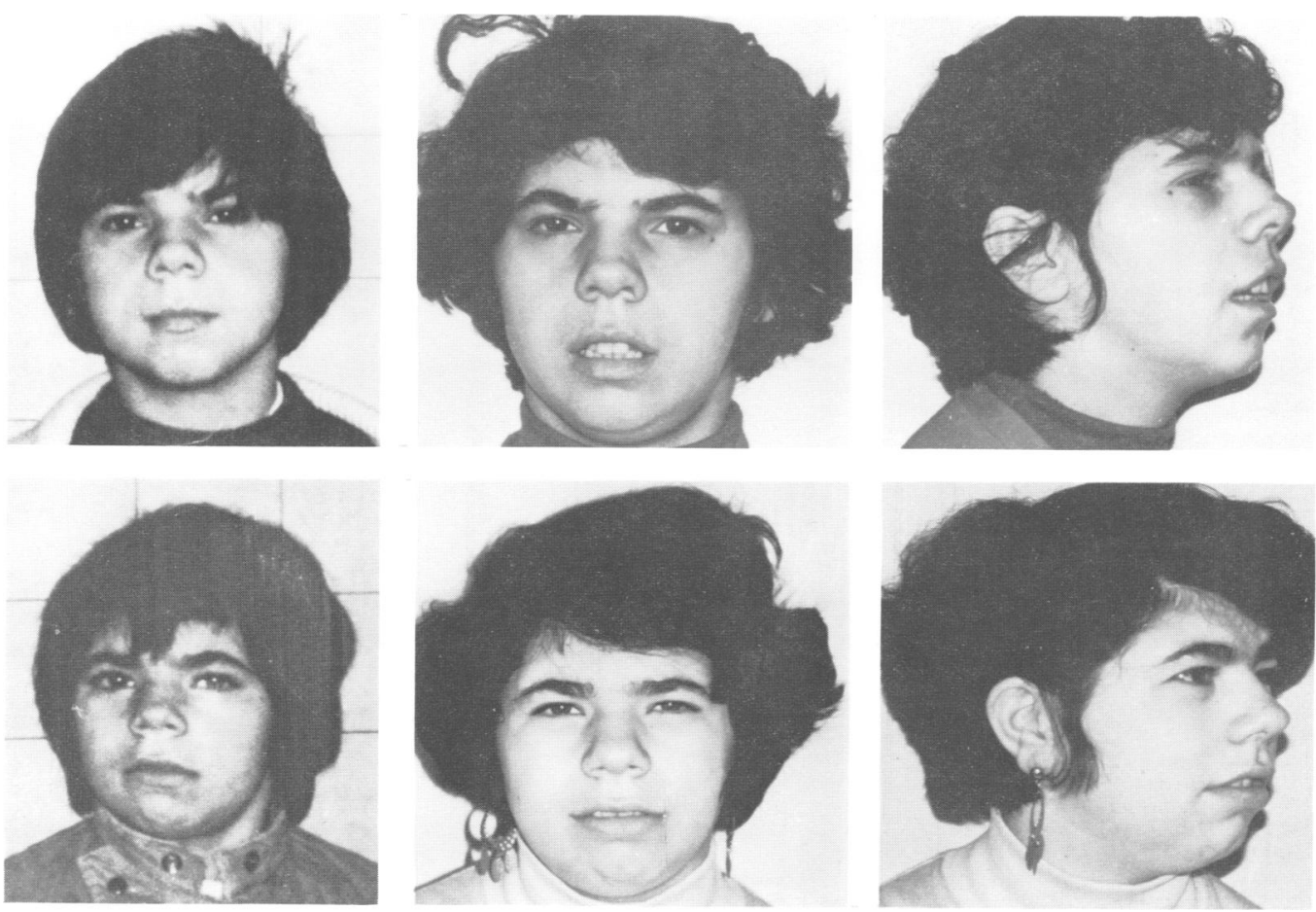
mandible, which increases in length and thickness resulting in the lower incisors overbiting the upper incisors. None of these bone changes nor prognathism were observed in the AFA syndrome subjects. Coarse and leathery skin with exaggerated pores and markings is characteristic of acromegaly, in which body hair and melanosis are also increased. However, the skin consistency is normal or doughy in persons with the AFA syndrome.

Thickened features and puffy appearance are also found in patients with full blown hypothyroidism. The myxoedema is characteristically swollen and not pitted, and it is most apparent around the eyes and on the dorsa of the hands and causes enlargement of the tongue. Persons with the AFA syndrome have some of the features of myxoedematous patients, although their skin is not pale, cool, and dry. Our patients had a general slowing of all intellectual functions, thick, slurred speech, and hoarseness. These features are also found in the myxoedematous patients.

The overlapping features of AFA patients and those with other related syndromes have been discussed in detail by Hughes et al. ${ }^{1}$ It is unclear if our family and the original AFA family have different conditions. The acromegaloid-myxoedematous facial appearance designation seems more appropriate for describing the present family.

1 Hughes HE, McAlpine PJ, Cox DW, Philipps S. An autosomal dominant syndrome with 'acromegaloid' features and mal dominant syndrome with 'acromegaloid' features
thickened oral mucosa. $\mathcal{F}$ Med Genet 1985;22:119-25. 